\title{
Transcriptomic analysis supports similar functional roles for the two thymuses of the tammar wallaby
}

\author{
Emily SW Wong ${ }^{1}$, Anthony T Papenfuss ${ }^{2}$, Andreas Heger ${ }^{3}$, Arthur L Hsu², Chris P Ponting ${ }^{3}$, Robert D Miller ${ }^{4}$, \\ Jane C Fenelon ${ }^{5}$, Marilyn B Renfree ${ }^{5}$, Richard A Gibbs ${ }^{6}$ and Katherine Belov ${ }^{1 *}$
}

\begin{abstract}
Background: The thymus plays a critical role in the development and maturation of T-cells. Humans have a single thoracic thymus and presence of a second thymus is considered an anomaly. However, many vertebrates have multiple thymuses. The tammar wallaby has two thymuses: a thoracic thymus (typically found in all mammals) and a dominant cervical thymus. Researchers have known about the presence of the two wallaby thymuses since the 1800s, but no genome-wide research has been carried out into possible functional differences between the two thymic tissues. Here, we used pyrosequencing to compare the transcriptomes of a cervical and thoracic thymus from a single 178 day old tammar wallaby.

Results: We show that both the tammar thoracic and the cervical thymuses displayed gene expression profiles consistent with roles in T-cell development. Both thymuses expressed genes that mediate distinct phases of T-cells differentiation, including the initial commitment of blood stem cells to the T-lineage, the generation of T-cell receptor diversity and development of thymic epithelial cells. Crucial immune genes, such as chemokines were also present. Comparable patterns of expression of non-coding RNAs were seen. 67 genes differentially expressed between the two thymuses were detected, and the possible significance of these results are discussed.

Conclusion: This is the first study comparing the transcriptomes of two thymuses from a single individual. Our finding supports that both thymuses are functionally equivalent and drive T-cell development. These results are an important first step in the understanding of the genetic processes that govern marsupial immunity, and also allow us to begin to trace the evolution of the mammalian immune system.
\end{abstract}

\section{Background}

The thymus is a site of T-cell differentiation and maturation. First discovered by Galen (130-200 AD), it is a primary lymphoid organ with a critical role in development of the immune system. Its major function is to eliminate developing $\mathrm{T}$-cells (thymoctyes) whose antigen receptor can bind self antigens and have the potential to cause autoimmune disease $[1,2]$. The thymus is comprised of two distinct regions, the cortex and the medulla, both of which produce thymic epithelial cells. On entering the thymus, thymocytes undergo clonal proliferation, lineage commitment and selection for a T-cell receptor (TCR)

\footnotetext{
* Correspondence: kathy.belov@sydney.edu.au

'Faculty of Veterinary Sciences, University of Sydney, Sydney, NSW 2006, Australia

Full list of author information is available at the end of the article
}

that can interact with self Major Histocompatibility Complex molecules. This is primarily under the control of cortical thymic epithelial cells [2]. To ensure that autoreactive thymocytes are eliminated, a diversity of selfantigens, representing a wide range of tissue types, is presented to thymocytes by medullary thymic epithelial cells [3]. Therefore the medullary epithelium must synthesize a large fraction of the potential proteome of the individual to display as self. Autoreactive thymocytes are eliminated by apoptosis. In total, $95-99 \%$ of thymocytes are eliminated this way, leaving only a $1-5 \%$ pool of functional $\mathrm{T}$-cells that eventually enter peripheral circulation as mature T-cells $[4,5]$.

All jawed vertebrates possess a thymus. Eutherian mammals, such as humans and dogs, usually possess a single thoracic thymus. A thymus in the neck of humans (called

\section{Biomed Central}


a cervical thymus) is considered to be an often asymptomatic pathological condition whereby the thoracic thymus has failed to descend to its proper mediastinal location $[6,7]$. However, the number of thymuses per animal, the anatomical position and structure of the thymic lobes and the exact developmental origin of the thymus all differ markedly among species [7]. Multiple thymuses are common in cold-blooded vertebrates, with five pairs found in shark, four in caecilian amphibians, three in salamanders and one found in cartilaginous fish [7]. In other eutherian mammals such as sheep, cattle, goat and horse, the paired thymuses consist of a distinct cervical and thoracic part; the stem of the thymus connecting the two sections typically disappears shortly after gestation $[8,9]$. Certain strains of mice possess an additional thymic organ in the cervical area, with distinct cortical and medullary regions that support positive and negative selection of thymocytes and export mature thymocytes to the circulation $[10,11]$.

The discovery of cervical thymuses in marsupials in the 1800 s provided some of the earliest documentation of the cervical thymus in any mammal [12-15]. It is now established that diprotodont marsupial species, including kangaroo, wallabies and possums, typically possess both cervical and thoracic thymuses [16-21]. The exception to this is the koala (Phascolarctos cinereus), in which a lone thoracic thymus is more commonly found [20]. Early studies focused on topological and anatomical descriptions of the thymuses including details on their organogenesis and relative growth rate $[12-15,18,19,22]$. Later, histological studies were used to determine the time of T-cell maturation [23-27].

Marsupials deliver highly altricial young that complete their development post-natally [28] so their two pairs of thymuses develop after birth while the young is in the pouch $[29,30]$, making them ideal model organisms to study the development of the immune system. At birth, marsupials lack functional lympoid tissues $[27,30,31]$ and humoral immune competence (mediated by antibodies). Cell-mediated immune responses (associated with T-cells) develop during pouch life (reviewed by [32]). In the tammar wallaby (Macropus eugenii), T-lymphocytes are observed at Day 2 in the cervical thymus [24] and $\mathrm{CD} 3+$ mature T-cells are first detected in thymic tissue on Day 12 postpartum [26]. This coincides with the differentiation of the cervical thymus into a rudimentary cortex and medulla $[24,26]$. The thymuses are largest from mid-pouch life, and adult lymphoid tissue structure is seen at approximately Day $120[24,26]$, which is why we chose 178 day old tissue for this experiment. At maturity, distinct cortical and medullary regions and Hassall's corpuscles, groups of specialized epithelial cells localized to the medulla that direct maturation of a lineage of T-regulatory cells, are present in both thymuses $[24,26,33]$.
The marsupial cervical thymus reaches enormous proportions during pouch life when it obscures all other cervical glands [17]. It is larger than the thoracic thymus at all development stages $[17,34]$. In a 150-day-old tammar wallaby pouch young the thoracic thymus weighs $94 \mathrm{mg}$, a mere $7 \%$ of the cervical thymus which weighs $1,400 \mathrm{mg}$ or $0.7 \%$ of total body weight [17]. At Day 120, the thoracic thymus is not as multi-lobed as earlier stages of the cervical thymus [24].

Several findings support the notion that both organs have a similar, if not identical role. Mature T-cells are present in both thymuses in the tammar and in another wallaby, the quokka (Setonix brachyurus) throughout development $[24,26]$. Onset of humoral responses are delayed upon removal of the cervical thymus and total thymectomy further delays, but does not abrogate, the ability of quokkas to mount a T-cell dependent humoral immune response [34]. Notably, neonatally thymectomized quokkas have a significantly reduced lifespan [35].

Here, we compare gene expression profiles in the cervical and thoracic thymus of a marsupial, the tammar wallaby. This is the first transcriptomic study of both the cervical and thoracic thymus from any species. We predicted that both organs would display similar transcriptomic profiles and express genes that are critical for thymic function.

\section{Results and discussion}

RNA from cervical and thoracic thymic tissues from a 178-day-old tammar wallaby pouch young was extracted and pyrosequenced using the Roche 454 platform. 758,062 reads with an average read length of 184 bases were generated. Checks on sequencing biases are provided as additional file 1 and additional file 2 . Annotated gene sequences are available at http://bioinf.wehi.edu.au/ tammar. Full datasets are stored in the NCBI Short Reads Archive, accessions SRX019250 and SRX019249.

\section{Gene abundance analysis}

Reads were aligned to the wallaby genome (version 1.0) using BLASTN. $87 \%$ of reads aligned to the genome. Reads that aligned well to two or more regions of the genome (28\%) were filtered out. This might have removed members of closely related gene families. Consequently, we also analysed reads without filtering. The two analyses resulted in similar results (data not shown) and had no effect on our main conclusions.

Based on alignment with the wallaby Ensembl gene build, 6,175 genes are expressed in either of the two thymuses, with 4,642 being expressed in both thymuses. However, as is commonly observed in transcriptome analyses, the majority (79\%) of reads failed to align to a predicted Ensembl gene. These reads are likely to represent $5^{\prime}$ or 3' untranslated regions (UTRs) not present in 
Ensembl gene models, or non-protein-coding transcripts or incorrectly predicted exons.

To increase the confidence of our analysis, we aligned the tammar reads against the higher quality opossum genome (a $7 \times$ coverage marsupial genome). A similar number of genes were identified in either of the two thymuses (6,060 genes). More reads aligned to intergenic sequence in the tammar assembly (79\%) compared with the opossum assembly (41\%). This may reflect the expression of regions poorly conserved between the two marsupial genomes and currently unannotated genes in tammar.

\section{Further thymic gene identification}

In order to improve the sensitivity of our sequence database searches, we assembled reads pooled from both tissues. Since most genes show similar expression levels in the two tissues (Figure 1), on average, this doubles the number of reads mapped to each gene (compared to separate assemblies) and thus will tend to provide longer transcript contigs. The resultant assembly of reads from both thymuses produced 36,591 transcript contigs. We used these contigs together with unassembled reads to identified 3,148 additional genes in the wallaby genome assembly.

Many immune genes are likely to be left unannotated by automated annotation pipelines, such as Ensembl, due to their more rapid evolution. To increase the sensitivity of immune gene identification we used manually curated opossum (Monodelphis domestica) immune gene lists $[36,37]$ of 1,549 opossum genes. Less than a third (463) of these were annotated by the Ensembl opossum geneset. Using this curated geneset we were able to identify the expression of an additional 222 immune genes in the wallaby, adding to the 356 expressed wallaby immune genes that were already annotated by Ensembl http:// bioinf.wehi.edu.au/tammar. We report the expression of 34 cytokines and their receptors (10 chemokines, 22 interleukins and 2 interferons), 22 natural killer cell receptors (20 Leukocyte Receptor Complex (LRC) genes and two Natural Killer Complex (NKC) genes), three antimicrobial peptides (two beta-defensins and one cathelicidin), post-switch immunoglobulin isotypes IgA and IgG and CD4 and CD8 T-cell markers. We expect

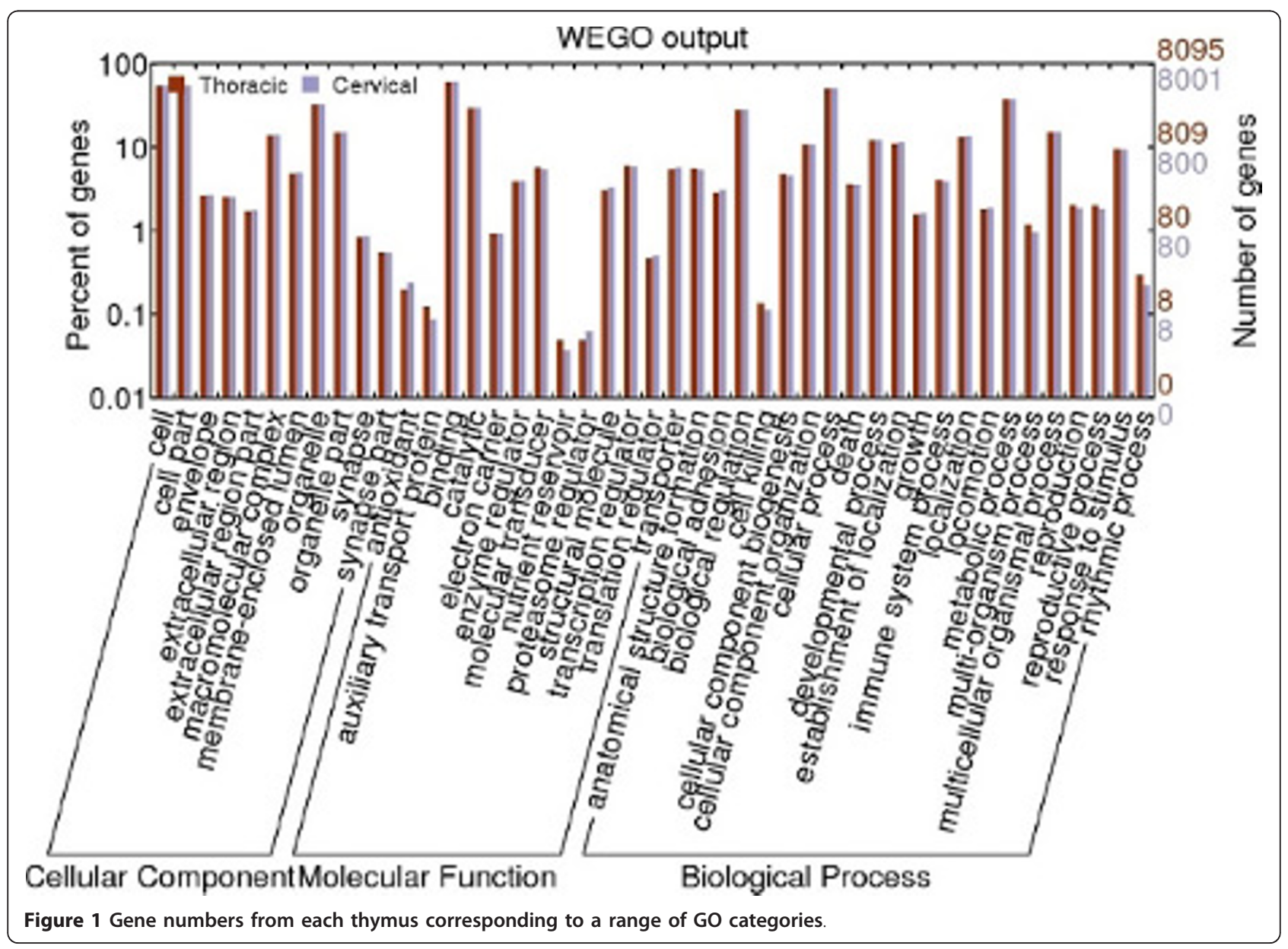


that unidentified immune genes are either unsequenced at this sequencing depth, are not expressed in the thymuses or are too divergent to identify using our search method.

\section{Thymic gene function}

We identified a total of 9,545 genes in the two tammar thymus pairs. Within this dataset, we identified transcription factors and signalling molecules that control the development of haematopoietic stem cells committed to T-cells lineages, including GATA3, IKZF1, RUNX3, LEF1, JAG1, NOTCH1, IL-7R, MYB, HEB and E2A (reviewed in [38]) (Table 1). These molecules are expressed during early T-cell development and are essential for the promotion of hematopoietic stem cells to the T-lineage. GATA3 and RUNX3 play important roles in CD4- and CD8-lineage choice, a process that occurs at later stages of T-cell development [38-42]. Together, these proteins form complex interactions which promote thymocyte differentiation. For example, $H E B$ and $E 2 A$ fall into a group of class I helix-loop-helix proteins, also known as E proteins. As heterodimers these activate the pre-T-cell antigen receptor alpha (PTCRA), an invariant T-cell receptor alpha chain that forms the pre-TCR essential for alpha-beta lineage $\mathrm{T}$-cell differentiation [43]. One of the two isoforms of $E 2 A, E 47$, also regulates the gene rearrangement proteins,

\begin{tabular}{l} 
Table $\mathbf{1}$ Human gene symbol and its corresponding \\
description of critical thymic genes transcribed in both \\
wallaby thymuses \\
\begin{tabular}{ll} 
HGNC & Description \\
Symbol & \\
\hline LTBR & Tumor necrosis factor receptor superfamily member 3 \\
\hline NOTCH1 & Transmembrane receptor for transcriptional activation \\
\hline JAG1 & NOTCH1 ligand \\
\hline IL7R & Interleukin-7 receptor subunit alpha precursor \\
\hline GATA3 & Trans-acting T-cell-specific transcription factor \\
\hline XRCC4 & DNA repair protein XRCC4 \\
\hline TRAF6 & TNF receptor-associated factor 6 \\
\hline RAG1 & V(D)J recombination-activating protein 1 \\
\hline RAG2 & V(D)J recombination-activating protein 2 \\
\hline LEF1 & Lymphoid enhancer-binding factor 1 \\
\hline IKZF1 & DNA-binding protein Ikaros \\
\hline RUNX3 & Runt-related transcription factor 3 \\
\hline DNTT & DNA nucleotidylexotransferase (Terminal addition \\
\hline EYB & Mybe) \\
\hline GFI1 & Zinc finger protein \\
\hline TP63 & Tumor protein 63 (p63) \\
\hline E2A & E-protein gene \\
\hline HEB &
\end{tabular} \\
\hline
\end{tabular}

$R A G 1 / 2$, which are initiators of $\mathrm{T}$-cell receptor gene recombination, a key step in the generation of receptor diversity, in early T-lymphocytes development $[43,44]$. Furthermore, E2A also acts in concert with Notch signalling, to promote differentiation of T-cells and to suppress progenitor cell commitment to NK and myeloid cell fates [45]. The Notch signalling pathway is a critical pathway for the initiation of $\mathrm{T}$ cell development [46-48]. Induced deletion of NOTCH1, a transmembrane transcription activator, in mouse results in an early stage blockage in T-cell development [47]. Notch signalling induced by Jagged1 (JAG1) plays a critical role in thymocyte cell-fate determination [49]. Notch signals have also been shown to cooperate with the $I L-7 R$ pathway to sustain $I L-7 R$ expression in proliferating thymocytes [50]. Signalling of $I L-7 R$ has a prominent role in thymocyte proliferation in early $\mathrm{T}$-cell development [51]. Accordingly, $I L-7 R$ is tightly regulated in later stage committed T-cells [52] and contributes to regulatory $\mathrm{T}$-cell development and homeostasis outside the thymus [53]. Notably, key genes involved in mouse cervical and thoracic thymopoiesis: RAG1, RAG2 and $D N T T$ [10], were identified in both tammar thymuses.

We detected the presence of key chemokine receptors, CCR7 and CCR9, in both wallaby thymuses. Chemokines are small proteins that are able to induce chemotaxis, and in the thymus, they help dictate the movement of $\mathrm{T}$-cells from initial precursor cell recruitment through to mature thymocyte export. Chemokine expression therefore must be controlled and this is reflected through the unique chemokine expression profiles in different thymic cell types [54]. Both CCR7 and CCR9 maintain an important role during $\mathrm{T}$-cell development, ensuring that developing thymocytes are positioned at specific thymic microenvironments to support T-cell differentiation [55]. Movement of thymocyte into the medulla from the cortex after positive selection is signalled by CCL7 [56], with premature positioning of $\mathrm{CD} 4+\mathrm{CD} 8+$ thymocytes into the medulla impairing T-cell development [57]. Similarly, CCL9 is responsible the movement of immature T-cells to the thymic subcapsular zone [58].

Other genes essential in thymocyte development include TRAF6, TP63 and LTBR. These genes are necessary for the development and maintenance of normal thymic architecture. TRAF6, a signal inducer of the NK-kB pathway, is necessary for the organization of medullary thymic epithelial cells (mTECs), and TP63 is required for normal epithelial development [59-61]. Cross-talk between mTECs and thymocytes is essential for mTEC differentiation. This exchange is mediated by the lymphotoxin beta receptor $(L T B R)$ [62].

All major classes of $\mathrm{T}$-cell receptor chains were expressed in both tammar thymuses (Table 2). The ability of $\mathrm{T}$-cells to recognize pathogens is mediated by their $\mathrm{T}$ cell receptors. $\mathrm{T}$-cell receptors have been detected in 
Table 2 Genomic locations in the tammar assembly and number of reads across both thymuses for constant regions of $\mathrm{T}$-cell receptors

\begin{tabular}{lll}
\hline & Genomic scaffold & Read count \\
\hline TRBC & 45360 & 85 \\
\hline 544743 & 30 \\
\hline TRDC & 148935 on chromosome $7 p$ & 25 \\
\hline TRAC & 32403 on chromosome $7 p$ & 2 \\
\hline TRGC & 6369 & 3 \\
\hline & 340996 & 1 \\
\hline TRMC & 21414 & 1 \\
\hline & 73733 & 1 \\
\hline & 92637 & 1 \\
\hline & 52643 & 6 \\
\hline
\end{tabular}

TRA/D have been mapped to chromosome 7p [74].

opossum neonates as early as Day 1 [63], although T-cell dependent cellular immune responses are not observed until the second week of development [64]. TCR chains are classified according to their constant regions-alpha (TRAC), beta $(T R B C)$, gamma (TRGC), delta (TRDC) and $\mathrm{mu}(T R M C)[65,66]$. The novel T-cell receptor mu (TRM) that was first discovered in marsupials and has not been found in eutherians $[66,67]$. In vertebrates, each T-cell bears a unique $\mathrm{T}$-cell receptor that is specific to a limited set of peptide and major histocompatibility complex (MHC) combinations [65]. The T-cell receptors (TCR) are formed from disulfide-linked heterodimers which are composed of either an alpha and a beta chain or a gamma and a delta chain. Alpha-beta $\mathrm{T}$-cell receptors recognize $\mathrm{MHC}$ molecules and undergo a process of ligand-driven positive and negative selection leading to maturation to T-helper cells or T-killer cells. Delta-gamma T-cells bind to a different set of ligands and do not require antigenbased selection for maturation $[68,69]$. To be effective in recognizing a wide variety of antigens, a diverse repertoire of TCR chains are generated through a process known as somatic recombination involving variable $(\mathrm{V})$, diversity(D) and joining $(\mathrm{J})$ genomic segments. Tammar transcripts displayed evidence of somatic recombination.

Four TCR beta chain constant (TRBC) genes showed highly variable number of transcripts (Table 2 ). This may be due to selective preference for certain $T R B C$ genes over others. Four TRBC genes exist in opossum, but both human and mouse TRB loci comprise of just two TRB constant genes $[67,70]$. We identified five putative TRBC genes in tammar genome, one of which we did not have any expression data for, and suggest that up to five TRB genes may potentially exist in tammar.
One TRDC transcript with 91\% identity with cloned wallaby TRDC transcript (Accession: AAP72021) was identified, with mismatches between the sequences likely to be due to either allelic differences or sequencing errors. The TRA/D locus spans $\geq 1 \mathrm{Mb}$ in the opossum, human and mouse genomes with the TRD locus nested within the TRA locus $[67,70]$. The presence of a single constant region for each chain is consistent with known TRA/D genomic structure in all vertebrates.

Two wallaby TRG constant regions (TRGC) genes were found. TRGC regions vary in number between species with human and mouse both possessing a single TRG locus which contains two and four TRGC genes, respectively, which are arranged in tandem cassettes $[71,72]$. The cow and sheep have two TRG loci each, which containing variable numbers of constant regions [73]. Only one TRG locus exists in the opossum, comprising a single constant gene, arranged in a translocon organization with $\mathrm{V}$ and $\mathrm{J}$ regions adjacent to one another [67]. Both scaffolds reside on chromosome 3p [74] but it remains to be determined whether the two wallaby TRGC genes localize to the same locus, like in human and mouse, or to different loci, as in ruminants. TRMC is also expressed at comparable levels between the two thymuses [66].

Both thymuses express transcripts involved in the process of somatic recombination (VDJ recombination) which is necessary for $\mathrm{T}$-cell receptor generation. These include recombination activating gene-1 (RAG1), RAG2, DNAdependent protein kinase, Artemis, DNA ligase IV and $X R C C 4$. The initiation of VDJ recombination involves the lymphoid-specific proteins, RAG1 and RAG2, which introduce double-strand breaks in signal sequence adjacent to coding segments (reviewed in [75]). Broken ends bind to DNA-dependent protein kinase which combines with Artemis to break the hairpin structure introduced by the RAG proteins [76,77]. Finally, the ligation of two ends is mediated by DNA ligase IV which forms a heteromultimer with $X R C C 4$, a protein that serves to enhance the joining activity [78-80].

Due to the low sequence coverage of the transcriptomes, the absence of some genes in our database was expected. We did not expect to see lowly expressed genes, and did not find thymic stromal lymphopoietin transcripts $(T S L P)$. In humans it promotes the expression of $C D 80$ and CD85 in dendritic cells, which in turn induce the expansion differentiation of groups of thymic T-cells into regulatory T-cells [33]. TSLP is expressed by Hassall's corpuscles, a distinct group of medullary TECs. Hassall's corpuscles are observed in the wallaby cervical and thoracic thymuses by days 21 and 30 respectively. Hence, we expected to identify TSLP in a 178 day old wallaby thymus. TSLP exhibit a high level of sequence divergence. We identified the gene using a hidden Markov model (HMM) profile, but not the transcript. 


\section{Non-protein-coding RNAs}

Given the abundance of transcripts that did not map to gene models, we performed some analyses on non-coding RNA transcripts identified in both thymuses. 3,188 putative non-coding contigs were conserved between the opossum and tammar wallaby. To identify putative non-coding RNAs (ncRNAs), all contigs were analysed using the program CPC [81]. Contigs were considered non-coding provided that they do not encode open reading frames and are not homologous to known protein sequences. 83,294 potential non-coding elements were identified. We subsequently aligned all potential non-coding RNAs to the opossum genome, resulting in 12,703 mapped contigs. To avoid the misannotation of UTRs as non-coding RNAs, we examined 3,524 contigs that aligned to a distance of $100 \mathrm{~kb}$ or greater to their adjacent Ensembl gene. Notably, approximately half of all predicted non-coding structural elements in human are located over $10 \mathrm{~kb}$ away from any known gene, although these have not been verified experimentally [82]. These contigs were searched against a comprehensive database of human UTR regions, UTRfull [83]. 3,188 reads remained after removal of sequences aligning to known UTRs with E-values $<10^{-3}$ The annotation of the resultant ncRNA candidates against non-coding databases, fRNAdb [84] and RFAM [85], identified 101 non-coding elements including 52 conserved non-coding structures that were determined by RNAz [86] and EvoFold [87] (Table 3). Wallaby non-coding contigs were located in the genome closest to genes that are involved in transcriptional regulation $\left(\mathrm{P}=4.3 \times 10^{-5}\right.$ Fisher exact test with Bonferroni correction). This is consistent with genomic observations in vertebrates, where conserved non-coding elements tend to be located adjacent to transcriptional regulators, suggesting distant regulatory functions [88-91]. Evolutionarily conserved non-coding elements show a level of synteny similar to coding genes [92], and behave as transcriptional enhancers in vivo $[91,93]$. Our results represent the first set of candidate ncRNAs conserved between marsupials. To examine whether any overlapping non-coding elements exist

Table 3 Classification of putative functional non-coding RNAs

\begin{tabular}{ll}
\hline NcRNA type & Number of NcRNAs identified \\
\hline piRNA & 72 \\
\hline tRNA & 16 \\
\hline miRNA & 13 \\
\hline Ribosomal RNA & 6 \\
\hline Unclassified small RNAs & 6 \\
\hline L1 LINE 3' elements & 2 \\
\hline RNAz non-coding structures & 28 \\
\hline EvoFold non-coding structures & 24 \\
\hline
\end{tabular}

The miRNAs map to seven separate seed regions. between our contigs and a highly conserved Fugu dataset [91], we searched against the conserved pufferfish sequences and retrieved four tammar contigs. Two of these were similar to piRNAs and one matched a known transposable L1 LINE 3' element.

\section{Differential gene expression between cervical and thoracic thymuses}

Of all genes identified in the two thymuses, only 67 genes were expressed at significantly different levels in the two thymuses ( $p<0.05$ Fisher exact test with FDR correction). The cervical thymus over-expressed a group of genes with strong associations to muscle structure, assembly and contraction. Testing for enrichment of GO terms in genes more highly expressed in the cervical thymus, identified nine over-represented terms $(p<0.05$; Fisher exact test with Bonferroni correction). Of these, eight GO terms were linked either directly or indirectly to muscle fibres $\left(p=2.8 \times 10^{-6}\right.$; Fisher exact test with Bonferroni correction) (Table 4; Figure 2). ATP2A1 together with ryanodine receptor, $R Y R 1$, and muscle glycogen phosphorylase (PYGM) are typically found in the sarcoplasmic reticulum, a subtype of smooth endoplasmic reticulum found in muscle fibres whose function is to store and release calcium ions. Both troponin $\mathrm{T}$ and creatine kinase muscle (CKM) were over-expressed by the cervical thymus. Notably, these genes are also highly expressed in human myoid cell lines [94].

Thymuses are known to carry cells containing striated myofibrils which closely resemble skeletal or cardiac muscle fibers. Known as myoid cells, these cells are conserved throughout vertebrate evolution, yet, prior to this study, myoid cells have not been identified in the marsupial thymus $[17,20,23,24,26,95]$. However, they have been described in vast numbers of eutherian, bird, reptile, amphibian, and teleost species [96-98]. Myoid cells appear to be abundant in non-eutherian mammals and younger eutherian animals. In birds and reptiles, however, they appear to increase in number with age. Reptilian myoid cells numbers vary depending on season

Table 4 Names of genes differentially expressed between the two thymuses linked to GO term 'muscle fibres'

\begin{tabular}{ll}
\hline Name & Gene symbol \\
\hline sarcoplasmic reticulum calcium ATPase 1 & ATP2A1 \\
\hline myosin binding protein C, slow type & MYBPC1 \\
\hline myosin regulatory light chain 2 & MYLF \\
\hline nebulin & NEB \\
\hline sarcoplasmic reticulum calcium release channel receptor & RYR1 \\
\hline fast skeletal muscle troponin C & TNNC2 \\
\hline slow skeletal muscle troponin T & TNNT1 \\
\hline fast skeletal muscle troponin T & TNNT3 \\
\hline
\end{tabular}




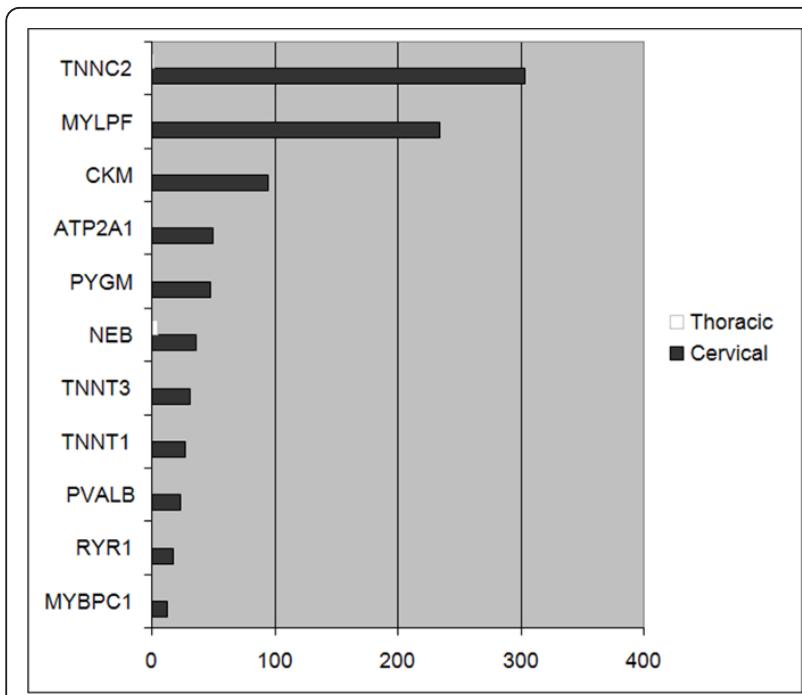

Figure 2 Read counts for differentially expressed muscle genes.

[96]. Furthermore, they display a wide spectrum of muscle cell developmental stages [96]. Although functional studies on myoid cells are lacking over all phyla, myoid cells have been shown in humans in vitro to have protective effects against apoptosis and to increase the population of CD4+ thymocytes [99]. In the medullary region of human embryos, there is an increase in the number of myoid cells along with a corresponding increase in amount of medullary myosin in the late developmental stages of the embryos [100]. It has been suggested that this may reflect the demands of the growing fetus for mature $\mathrm{T}$ lymphocytes [100]. If this is true, it is conceivable that such a demand may be heightened by the immunologically immature state of marsupials at birth.

Several genes, identified in the cervical thymus, are integral to muscle contraction. Given that myoid cells are known to spontaneously contract in vitro this may aid the movement of thymocytes in vivo $[100,101]$. The $\mathrm{Ca}^{2+}$ transporter molecule, $A T P 2 A$, and the troponin proteins, are involved in the $\mathrm{Ca}^{2+}$ binding pathway that leads to muscle contraction $[102,103]$. These were over-expressed in the cervical thymus.

The preponderance of muscle related genes in the cervical thymus is unlikely to be due to the expression of a diverse array of tissue-specific genes in medullary thymic epithelial cells (mTECs) during negative selection [104] even though numerous tissue-specific antigens are known to be expressed in mTEC cells. The expression of tissue specific antigens allows potential auto-immune $\mathrm{T}$ cells to be detected and subsequently destroyed. Promiscuously expressed tissue-specific antigens tend to be colocalized in genomic clusters and their expression appears to be primarily controlled by pathways involving the transcriptional regulator, AIRE $[105,106]$. But the differentially expressed muscle genes in tammar thymuses do not appear to be clustered in the genome (based on the opossum genome) and it is unlikely that a muscle-specific group of genes would be expressed in a concerted fashion by mTECs in one thymus but not the other.

Parvalbumin alpha (PVALB) was also expressed more highly in the cervical than in the thoracic thymus. PVALB is a calcium-binding protein found in skeletal myofibrils and GABA-producing neurons, with a role in the relaxation of muscle after contraction $[107,108]$. Interestingly, a paralog of $P V A L B$, avian thymic hormone $(A T H)$, promotes chicken thymocyte maturation $[109,110]$. According to Ensembl annotations, orthologs of $A T H$ are present in marsupials and monotremes but are missing in eutherian species. This not only suggests that $A T H$ was lost in the eutherian lineage but also indicates a possible role of $A T H$ in T-cell development in marsupials and monotremes, as in chicken. However, transcripts of $A T H$ were not detected in either tammar thymus transcriptome, probably due to the low coverage of the transcriptome. Tammar oncomoulin $(O C M)$ transcripts were detected in the thoracic thymus. OCM is also a paralog of PVALB and functions as an oncodevelopmental protein in human. Although human oncomodulin is mainly expressed in early embryonic cells, chicken oncomodulin-like protein expression is largely localized to the thymus [111]. Further studies are required to ascertain whether wallaby $O C M$ is expressed more broadly and to determine whether it has an immunomodulary role in the thymus. $A T H$ and $O C M$ are located in close proximity of one another and show conserved synteny in chicken and opossum genomes. Their genomic position remains to be determined in the tammar due to short contig lengths.

\section{Possible explanations for differential expression}

Differences in gene expression between the two thymuses may be explained in several ways. Firstly, differences may merely be reflective of uneven sequencing of thymic compartments. Thymic anatomical microenvironments are unique and molecular gradients control T-cell development [112]. Eutherian myoid cells localize in the thymic medulla, although occasional expression in the corticomedullary junction has been observed [113,114]. Although we have sequenced equivalent amounts of cDNA from both thymuses, it is possible that more thymic epithelial cells and thymocytes were harvested in the thoracic thymus, whilst a greater number of myoid cells were sampled in the cervical. Yet, we cannot dismiss that the cervical thymus may indeed contain a greater proportion of myoid cells than the thoracic thymus. In human, a correlation exists between the abundance of myoid cells with fetal development time [100]. In marsupials, this is supported 
by the fact that the cervical thymus reaches functional maturity earlier than the thoracic which may result in differential counts of myoid cells. We did not observe other variations in expression that could clearly be explained by developmental differences between the two thymuses. The higher expression of certain T-cell developmentally-associated genes in the thoracic thymus (CD74, CD3G and NOTCH1) may be indicative of some differences in function between the thymuses. However, this is highly speculative at this early stage as sampling bias cannot be ruled out.

\section{Tissue-specific antigens}

Variation between tissue-restricted antigens expressed by multiple thymuses presents a potential susceptibility factor for autoimmunity. In mouse, cervical and thoracic thymuses have shown to express varying amounts of selfantigens [11]. In light of this, we compared 211 genes determined to be regulated by Aire in mouse medullary thymic epithelial cells [115] to tammar gene expression. In human and mouse, Aire regulates autoimmunity by promoting the expression of a range of tissue-restricted antigens in medullary epithelial cells.

Similar levels of self-antigens are expressed by the two tammar thymuses. Only one tissue-specific antigen, collagen type 1A (COL1A1), was regulated by Aire in mouse medullary thymic epithelial cells [116] and was differentially expressed between the two wallaby thymuses. However, given that our data represent a collection of all thymic cell types, COL1A1 may not be predominantly expressed by medullary epithelial cells. COL1A1 is a major component of muscle fibres [117]. In the context of differential co-expression of other muscle genes, variation of COL1A1 expression may more likely be attributed to myoid cell differences rather than selection for selftolerance by medullary epithelial cells.

\section{Summary}

Our results indicate that both marsupial thymuses are largely functionally equivalent. Both thymuses expressed genes that support thymic differentiation and function. These include transcripts encoding proteins with critical roles in directing thymic environment development (e.g. TRAF6, TP63 and LTBR) and T-cell lineage differentiation (e.g. IL-7R, NOTCH1, GATA3, SPI1, IKZF1). All T-cell receptors were expressed. In addition, key genes involved in mouse cervical and thoracic thymopoiesis were identified in both tammar wallaby thymuses. A relatively small number of genes were differentially expressed between the thymuses but these differences may be attributed to sampling artefacts. Notably, gene networks of known thymic function were not differentially expressed. The highly similar transcriptomic landscape of both tammar thymuses suggests that multiple thymuses in other species are also likely to show similar patterns of gene expression.

We have shown that both wallaby thymuses express similar levels of self-antigens. It is feasible that presence of multiple thymuses differentially selecting for self-tolerant $\mathrm{T}$ cells may result in autoimmunity if the two organs are projecting different versions of self [11]. In the mouse, variability in autoantigen expression has been reported [11], but we did not detect any differences in self-antigen expression between the two thymuses in the tammar wallaby.

The presence of a cervical thymus in the wallaby may be an evolutionary modification that allows rapid post-natal development of immunocompetence. Wallabies are born without immunological tissues and need to develop an immune system quickly to survive pathogen challenge in a closed pouch [27]. The size of the thoracic thymus is restricted by the size of the ribcage, while the thymus in the neck is able to grow unrestricted and allows earlier development of functional T-cells [118].

\section{Conclusion}

This is the first study to compare gene expression in the two thymuses of a marsupial, or in fact, any species. Both tammar wallaby thymuses have largely identical roles in T-cell development and maturation and possess basic thymic functions that are comparable to human and mouse. Crucial genes involved in T-cell differentiation, positive and negative selection of T-cells, immature $\mathrm{T}$-cell proliferation, as well as $\mathrm{T}$-cell receptor signalling are present. Our findings provide a springboard for further research into the development of the marsupial immune system and the evolution of the mammalian immune system.

\section{Methods}

\section{Library construction and sequencing}

Tissues from both thymuses were harvested from a 178 day-old tammar wallaby pouch young of Kangaroo Island, South Australian origin that was killed for another project approved by the University of Melbourne Animal Experimentation Ethics Committees. cDNA libraries were generated from the samples and sequenced at the Human Genome Sequencing Center at the Baylor College of Medicine, Houston, Texas, USA, using the Roche 454 Genome Sequencer FLX System. Two runs were completed with each run comprising of half runs of each thymus.

\section{Reads analysis}

Individual reads were assigned to gene models based on both the Ensembl wallaby and opossum genesets (Release 56). The opossum geneset is the highest quality genome of any marsupial to date and provides a useful quality 
comparison to the low coverage wallaby genome. A similar numbers of protein-coding genes are predicted by Ensembl between the two dataset $(15,280$ in wallaby and 19,466 in opossum). Reads were filtered and aligned to the Macropus eugenii 1.0 assembly using GMAP [119] and to the Monodelphis domestica (Mondom5) genome assembly using GMAP [119] and BLASTN [120]. The filtering stage involves removing reads mapping to more than one area with $<10 \%$ difference in scores, removing reads with less than 30 bases aligning and those with alignment coverage $<90 \%$.

\section{Gene abundance analysis}

Ensembl annotations were used to assign counts to reads aligned against the genomes. We considered reads that align with less than $5 \%$ of the sequence to an exon to be intergenic. Curated immune gene lists derived from Belov et al. 2007 [36] and Wong et al. 2006 [37] were specially used to derive read counts for immune genes. Fisher's exact test was used to test for differential expression between the two thymuses using the $\mathrm{R}$ statistical language [121]. Benjamini-Hochberg's method (FDR) [122] was used to correct for multiple testing.

Functional term enrichment analyses were performed using DAVID [123] and Ontologizer [124]. To obtain a more careful in depth look at genes of interest, we searched reads mapping to particular genes against the NCBI nr databases and also examined the genomic positions of reads using the UCSC genome browser by adding custom tracks to the opossum assembly.

\section{Targeted gene search}

To check the presence of gene expression for genes that may have been excluded by the stringent filtering procedure, we used a less conservative method of removing reads of low quality. This involves the stripping of vectors and 454 adapters and excising repeat regions identified by RepeatMasker [125]. Reads were assembled using CAP3 [126]. Contigs aligning with E-value $<10^{-5}$ were assigned to Ensembl gene models. Common genes between this gene list, the curated immune genes and genes identified for abundance analysis were determined by comparing gene symbols.

\section{Non-coding genes}

Coding potentials of assembled transcripts were assessed using CPC [81]. Reads aligning < $100 \mathrm{~kb}$ from opossum Ensembl genes in the genome were removed. Potential non-coding contigs were searched using blastn against the UTRfull database, a comprehensive collection of human UTR sequences curated by UTRdb [83]. Those matching with E-values $<10^{-3}$ were removed from the pool of potential nrRNA candidates. Remaining sequences were annotated by searching fRNAdb [84] using blastn (E-value cut-off $<10^{-3}$ ) and searching against the Rfam [85] covariance models.

\section{Tissue-specific antigens}

Genes determined to be regulated by Aire in mouse medullary thymic epithelial cells from Affymetrix microarrays (Mouse 430 2.0) [115] were compared to wallaby genes. Genes that were significantly differentially expressed between the two thymuses were analysed.

\section{Additional material}

Additional file 1: MA plot. Average count of a gene between the two thymuses is shown on the $\mathrm{x}$-axis and the count difference between the two thymuses for the same gene is shown on the $y$-axis. No obvious outliers are observed.

Additional file 2: Graph examining gene length bias using Ensembl opossum gene models. Length of gene is shown on the $x$-axis and $\log _{10}(P$-value $)$ on the $y$-axis. No length bias is evident.

\section{Acknowledgements}

We thank Assoc Prof Geoff Shaw for assistance in collection of the tammar thymuses and Helen Clark for RNA extraction. EW was supported by an ARC Centre of Kangaroo Genomics and Jean Walker Faculty Scholarship. The research in the CPP group was supported financially by the UK MRC and BBSRC. MBR was supported by an Australian Research Council Federation Fellowship. KB was supported by a University of Sydney Thompson fellowship.

\section{Author details}

${ }^{1}$ Faculty of Veterinary Sciences, University of Sydney, Sydney, NSW 2006, Australia. ${ }^{2}$ Bioinformatics Division, The Walter and Eliza Hall Institute for Medical Research, Parkville, Victoria 3050, Australia. ${ }^{3}$ Medical Research Council Functional Genomics Unit, Department of Physiology, Anatomy and Genetics, University of Oxford, Oxford, UK. ${ }^{4}$ Center for Evolutionary and Theoretical Immunology, Department of Biology, University of New Mexico, Albuquerque, NM, USA. ${ }^{5}$ ARC Centre of Excellence in Kangaroo Genomics, Department of Zoology, University of Melbourne, Victoria 3010, Australia. ${ }^{6}$ Department of Molecular and Human Genetics, Human Genome Sequencing Center, Baylor College of Medicine, One Baylor Plaza, Houston, TX 77030, USA.

\section{Authors' contributions}

EW performed the analyses, participated in the design of the study and wrote the manuscript. AP designed scripts used for read analyses and participated in study design. AH designed and ran scripts for read analyses. ALH performed the assembly of reads. MR supplied the animals and advised on the study and revised the manuscript. CP advised on the study and revised the manuscript. JF collected the samples. RM advised on the study and revised the manuscript. RG provided sequencing technology and advised on the study. KB conceived of the study, participated in study design and revised drafts of the manuscripts. All authors approved the final manuscript.

Received: 11 February 2010 Accepted: 19 August 2011 Published: 19 August 2011

\section{References}

1. Miller JFAP: Effect of neonatal thymectomy on the immunlogical responsiveness of the mouse. Proc Roy Soc 1962, 156B:410-428.

2. Ribatti D, Crivellato $E$, Vacca A: Miller's seminal studies on the role of thymus in immunity. Clinical and Experimental Immunology 2006, 144(3):371-375.

3. Magalhães DAR, Silveira ELV, Junta CM, Sandrin-Garcia P, Fachin AL, Donadi EA, Sakamoto-Hojo ET, Passos GAS: Promiscuous gene expression 
in the thymus: the root of central tolerance. Clinical \& Developmental Immunology 2006, 13(2-4):81-99.

4. Scollay RG, Butcher EC, Weissman IL: Thymus cell migration. Quantitative aspects of cellular traffic from the thymus to the periphery in mice. Eur $J$ Immunol 1980, 10(3):210-218.

5. Egerton M, Scollay R, Shortman K: Kinetics of mature T-cell development in the thymus. Proc Natl Acad Sci USA 1990, 87(7):2579-2582.

6. Sturm-O'Brien AK, Salazar JD, Byrd RH, Popek EJ, Giannoni CM, Friedman EM, Sulek M, Larrier DR: Cervical thymic anomalies-the Texas Children's Hospital experience. Laryngoscope 2009, 119(10):1988-1993.

7. Rodewald HR: Thymus organogenesis. Annu Rev Immunol 2008, 26:355-388.

8. Jordan RK: Development of sheep thymus in relation to in utero thymectomy experiments. Eur J Immunol 1976, 6(10):693-698.

9. Park EA: Extirpation of the Thymus in the Guinea Pig. J Exp Med 1917, 25(1):129-152

10. Terszowski G, Muller SM, Bleul CC, Blum C, Schirmbeck R, Reimann J, Du Pasquier L, Amagai T, Boehm T, Rodewald HR: Evidence for a functional second thymus in mice. Science 2006, 312(5771):284-287.

11. Dooley J, Erickson M, Gillard GO, Farr AG: Cervical thymus in the mouse. Journal of Immunology (Baltimore, Md: 1950) 2006, 176(11):6484-6490.

12. Symington J: The Thymus Gland in the Marsupialia. J Anat Physiol 1898, 32(Pt 2):278-291.

13. Symington J: A note on the thymus gland in the koala (Phascolarctos cinereus). J Anat Physiol 1900, 34:226-227.

14. Johnstone J: Cervical glands of marsupials. Proc Trans Lpool Biol Soc 1901, 15:354-362.

15. Johnstone J: The thymus in the marsupials. J Linn Soc (Zool) 1898, 26:537-557.

16. Sonntag C: Contributions to the visceral anatomy and myology of the marsupial. Proc Zool Soc Lond 1921, 91:851-882.

17. Yadav M: The presence of the cervical and thoracic thymus lobes in marsupials. Aust J Zool 1973, 21(3):285-301.

18. Fraser $E$ : The development of the thymus, epithelial bodies and the thyroid in the Marsupialia. Phil Trans R Soc Ser B 1915, 207:87-112.

19. Fraser $E$, Hill J: The development of the thymus epithelial bodies and thyroid in Trichosurus vulpecula. Phil Trans R Soc Ser B 1915, 207:1-85.

20. Haynes J: The marsupial and monotreme thymus, revisited. Journal of Zoology, London 2001, 253:167-173.

21. Windle B, Parson F: On the anatomy of Macropus rufus. I Anat Physiolo Lond 1898, 32:119-134

22. Yadav M, Stanley NF, Waring H: The thymus glands of a marsupial, Setonix brachyurus (quokka), and their role in immune responses. Structure and growth of the thymus glands. The Australian Journal of Experimental Biology and Medical Science 1972, 50(3):347-356.

23. Canfield P, Hemsley S, Connolly J: Histological and immunohistological study of the developing and involuting superficial cervical thymus in the koala (Phascolarctos cinereus). Journal of Anatomy 1996, 189(Pt 1):159-169.

24. Basden K, Cooper DW, Deane EM: Development of the lymphoid tissues of the tammar wallaby Macropus eugenii. Reproduction, Fertility, and Development 1997, 9(2):243-254.

25. Old JM, Deane EM: Immunohistochemistry of the lymphoid tissues of the tammar wallaby, Macropus eugenii. J Anat 2002, 201(3):257-266.

26. Old JM, Deane EM: The detection of mature $\mathrm{T}$ - and B-cells during development of the lymphoid tissues of the tammar wallaby (Macropus eugenii). Journal of Anatomy 2003, 203(1):123-131.

27. Old JM, Deane EM: Development of the immune system and immunological protection in marsupial pouch young. Developmental \& Comparative Immunology 2000, 24(5):445-454.

28. Renfree MB: Society for Reproductive Biology Founders' Lecture 2006-life in the pouch: womb with a view. Reprod Fertil Dev 2006, 18(7):721-734.

29. Soloman JB: Foetal and neonatal immunology. Amsterdam: North-Holland Pub. Co.; 1971

30. Tyndale-Biscoe CH, Renfree M: Reproductive Physiology of Marsupials. New York: Cambridge University Press; 1987.

31. Nelson JE: Developmental staging in a marsupial Dasyurus hallucatus. Anat Embryol (Berl) 1992, 185(4):335-354

32. Ashman R, Keast D, Stanley NF, Waring $H$ : The Immunological Responses of Marsupials. Amer Zool 1975, 15(1):155-166.
33. Watanabe N, Wang YH, Lee HK, Ito T, Cao W, Liu YJ: Hassall's corpuscles instruct dendritic cells to induce $C D 4+C D 25+$ regulatory $T$ cells in human thymus. Nature 2005, 436(7054):1181-1185.

34. Stanley NF, Yadav M, Waring $H$, Eadie M: The effect of thymectomy on response to various antigens of a marsupial Setonix brachyurus (quokka). Aust J Exp Biol Med 1972, 50(6):689-702.

35. Ashman RB, Waring $H$, Stanley NF: Adult mortality after neonatal thymectomy in a marsupial, Setonix brachyurus (quokka). Proceedings of the Society for Experimental Biology and Medicine Society for Experimental Biology and Medicine (New York, NY) 1973, 144(3):819-821.

36. Belov K, Sanderson CE, Deakin JE, Wong ES, Assange D, McColl KA, Gout A, de Bono B, Barrow AD, Speed TP, et al: Characterization of the opossum immune genome provides insights into the evolution of the mammalian immune system. Genome Res 2007, 17(7):982-991.

37. Wong E, Young L, Papenfuss A, Belov K: In silico identification of opossum cytokine genes suggests the complexity of the marsupial immune system rivals that of eutherian mammals. Immunome Research 2006, 2(1):4-4.

38. Rothenberg EV, Scripture-Adams DD: Competition and collaboration: GATA-3, PU.1, and Notch signaling in early T-cell fate determination. Semin Immunol 2008, 20(4):236-246.

39. Taniuchi I, Osato M, Egawa T, Sunshine MJ, Bae SC, Komori T, Ito Y, Littman DR: Differential requirements for Runx proteins in CD4 repression and epigenetic silencing during $\mathrm{T}$ lymphocyte development. Cell 2002, 111(5):621-633.

40. Woolf E, Xiao C, Fainaru O, Lotem J, Rosen D, Negreanu V, Bernstein Y, Goldenberg D, Brenner O, Berke G, et al: Runx3 and Runx1 are required for CD8 T cell development during thymopoiesis. Proceedings of the National Academy of Sciences of the United States of America 2003, 100(13):7731-7736

41. Nawijn MC, Ferreira R, Dingjan GM, Kahre O, Drabek D, Karis A, Grosveld F, Hendriks RW: Enforced expression of GATA-3 during T cell development inhibits maturation of CD8 single-positive cells and induces thymic lymphoma in transgenic mice. Journal of Immunology (Baltimore, Md: 1950) 2001, 167(2):715-723.

42. Hendriks RW, Nawijn MC, Engel JD, van Doorninck H, Grosveld F, Karis A: Expression of the transcription factor GATA-3 is required for the development of the earliest T cell progenitors and correlates with stages of cellular proliferation in the thymus. European Journal of Immunology 1999, 29(6):1912-1918.

43. Tremblay M, Herblot S, Lecuyer E, Hoang T: Regulation of $\mathrm{pT}$ alpha gene expression by a dosage of E2A, HEB, and SCL. J Biol Chem 2003, 278(15):12680-12687.

44. Schlissel M, Voronova A, Baltimore D: Helix-loop-helix transcription factor E47 activates germ-line immunoglobulin heavy-chain gene transcription and rearrangement in a pre-T-cell line. Genes Dev 1991, 5(8):1367-1376.

45. Ikawa T, Kawamoto H, Goldrath AW, Murre C: E proteins and Notch signaling cooperate to promote $\mathrm{T}$ cell lineage specification and commitment. J Exp Med 2006, 203(5):1329-1342.

46. Tanigaki K, Honjo T: Regulation of lymphocyte development by Notch signaling. Nature Immunology 2007, 8(5):451-456.

47. Radtke F, Wilson A, Mancini SJC, MacDonald HR: Notch regulation of lymphocyte development and function. Nature Immunology 2004, 5(3):247-253

48. Hozumi K, Mailhos C, Negishi N, Hirano K-i, Yahata T, Ando K, Zuklys S, Holländer GA, Shima DT, Habu S: Delta-like 4 is indispensable in thymic environment specific for T cell development. The Journal of Experimental Medicine 2008, 205(11):2507-2513.

49. Jiménez E, Vicente A, Sacedón R, Muñoz JJ, Weinmaster G, Zapata AG, Varas A: Distinct Mechanisms Contribute to Generate and Change the CD4:CD8 Cell Ratio During Thymus Development: A Role for the Notch Ligand, Jagged1. The Journal of Immunology 2001, 166:5898-5908.

50. Garcia-Peydro M, de Yebenes VG, Toribio ML: Notch1 and IL-7 receptor interplay maintains proliferation of human thymic progenitors while suppressing non-T cell fates. J Immunol 2006, 177(6):3711-3720.

51. Peschon JJ, Morrissey PJ, Grabstein KH, Ramsdell FJ, Maraskovsky E, Gliniak BC, Park LS, Ziegler SF, Williams DE, Ware CB, et al: Early lymphocyte expansion is severely impaired in interleukin 7 receptordeficient mice. J Exp Med 1994, 180(5):1955-1960. 
52. $Y u Q$, Erman $B$, Park $J H$, Feigenbaum $L$, Singer A: IL-7 receptor signals inhibit expression of transcription factors TCF-1, LEF-1, and RORgammat: impact on thymocyte development. J Exp Med 2004, 200(6):797-803.

53. Bayer $A L$, Lee $J Y$, de la Barrera $A$, Surh $C D$, Malek TR: A function for IL-7R for CD4+CD25+Foxp3+ T regulatory cells. J Immunol 2008, 181(1):225-234.

54. Wolf SS, Cohen A: Expression of cytokines and their receptors by human thymocytes and thymic stromal cells. Immunology 1992, 77(3):362-368.

55. Uehara S, Song K, Farber JM, Love PE: Characterization of CCR9 expression and CCL25/thymus-expressed chemokine responsiveness during $\mathrm{T}$ cell development: CD3(high)CD69+ thymocytes and gammadeltaTCR+ thymocytes preferentially respond to CCL25. J Immunol 2002, 168(1):134-142.

56. Ueno T, Saito F, Gray DH, Kuse S, Hieshima K, Nakano H, Kakiuchi T, Lipp M, Boyd RL, Takahama Y: CCR7 signals are essential for cortex-medulla migration of developing thymocytes. J Exp Med 2004, 200(4):493-505.

57. Kwan J, Killeen N: CCR7 directs the migration of thymocytes into the thymic medulla. J Immunol 2004, 172(7):3999-4007.

58. Benz C, Heinzel K, Bleul CC: Homing of immature thymocytes to the subcapsular microenvironment within the thymus is not an absolute requirement for T cell development. Eur J Immunol 2004, 34(12):3652-3663.

59. Senoo M, Pinto F, Crum CP, McKeon F: p63 Is essential for the proliferative potential of stem cells in stratified epithelia. Cell 2007, 129(3):523-536.

60. Dickson KM, Bhakar AL, Barker PA: TRAF6-dependent NF-kB transcriptional activity during mouse development. Developmental Dynamics: An Official Publication of the American Association of Anatomists 2004, 231(1):122-127.

61. Akiyama T, Maeda S, Yamane S, Ogino K, Kasai M, Kajiura F, Matsumoto M Inoue J-i: Dependence of self-tolerance on TRAF6-directed development of thymic stroma. Science (New York, NY) 2005, 308(5719):248-251.

62. Boehm T, Scheu S, Pfeffer K, Bleul CC: Thymic medullary epithelial cell differentiation, thymocyte emigration, and the control of autoimmunity require lympho-epithelial cross talk via LTbetaR. The Journal of Experimental Medicine 2003, 198(5):757-769.

63. Parra ZE, Baker ML, Lopez AM, Trujillo J, Volpe JM, Miller RD: TCR mu recombination and transcription relative to the conventional TCR during postnatal development in opossums. J Immunol 2009, 182(1):154-163.

64. LaPlante ES, Burrell R, Watne AL, Taylor DL, Zimmermann B: Skin allograft studies in the pouch young of the opossum. Transplantation 1969, 7(1):67-72

65. Janeway CA, Travers P, Walport M, Shlomchik M: Immunobiology. New York: Garland Publishing; 52001.

66. Parra ZE, Baker ML, Schwarz RS, Deakin JE, Lindblad-Toh K, Miller RD: A unique T cell receptor discovered in marsupials. Proc Natl Acad Sci USA 2007, 104(23):9776-9781.

67. Parra ZE, Baker ML, Hathaway J, Lopez AM, Trujillo J, Sharp A, Miller RD: Comparative genomic analysis and evolution of the $T$ cell receptor loci in the opossum Monodelphis domestica. BMC Genomics 2008, 9:111.

68. Jensen KD, Su X, Shin S, Li L, Youssef S, Yamasaki S, Steinman L, Saito T, Locksley RM, Davis MM, et al: Thymic selection determines gammadelta $T$ cell effector fate: antigen-naive cells make interleukin-17 and antigenexperienced cells make interferon gamma. Immunity 2008, 29(1):90-100.

69. Chien $\mathrm{YH}$, Konigshofer $\mathrm{Y}$ : Antigen recognition by gammadelta T cells. Immunol Rev 2007, 215:46-58.

70. Glusman G, Rowen L, Lee I, Boysen C, Roach JC, Smit AF, Wang K, Koop BF, Hood L: Comparative genomics of the human and mouse $T$ cell receptor loci. Immunity 2001, 15(3):337-349.

71. Vernooij BT, Lenstra JA, Wang K, Hood L: Organization of the murine T-cell receptor gamma locus. Genomics 1993, 17(3):566-574.

72. Lefranc MP, Rabbitts TH: Two tandemly organized human genes encoding the T-cell gamma constant-region sequences show multiple rearrangement in different T-cell types. Nature 1985, 316(6027):464-466.

73. Vaccarelli G, Miccoli MC, Antonacci R, Pesole G, Ciccarese S: Genomic organization and recombinational unit duplication-driven evolution of ovine and bovine T cell receptor gamma loci. BMC Genomics 2008, 9:81.

74. Sanderson CE, Belov K, Deakin JE: Physical Mapping of Immune Genes in the Tammar Wallaby (Macropus eugenii). Cytogenet Genome Res 2009, 127(1):21-5

75. Gellert M: V(D)J recombination: RAG proteins, repair factors, and regulation. Annu Rev Biochem 2002, 71:101-132.
76. Hammarsten O, Chu G: DNA-dependent protein kinase: DNA binding and activation in the absence of Ku. Proc Natl Acad Sci USA 1998, 95(2):525-530

77. Ma Y, Pannicke U, Schwarz K, Lieber MR: Hairpin opening and overhang processing by an Artemis/DNA-dependent protein kinase complex in nonhomologous end joining and V(D)J recombination. Cell 2002, 108(6):781-794.

78. Grawunder U, Wilm M, Wu X, Kulesza P, Wilson TE, Mann M, Lieber MR: Activity of DNA ligase IV stimulated by complex formation with XRCC4 protein in mammalian cells. Nature 1997, 388(6641):492-495.

79. Grawunder U, Zimmer D, Fugmann S, Schwarz K, Lieber MR: DNA ligase IV is essential for $V(D) J$ recombination and DNA double-strand break repair in human precursor lymphocytes. Mol Cell 1998, 2(4):477-484.

80. Modesti M, Hesse JE, Gellert M: DNA binding of Xrcc4 protein is associated with $\mathrm{V}(\mathrm{D}) \mathrm{J}$ recombination but not with stimulation of DNA ligase IV activity. EMBO J 1999, 18(7):2008-2018.

81. Kong L, Zhang Y, Ye ZQ, Liu XQ, Zhao SQ, Wei L, Gao G: CPC: assess the protein-coding potential of transcripts using sequence features and support vector machine. Nucleic Acids Res 2007, , 35 Web Server: W345-349.

82. Washietl S, Hofacker IL, Lukasser M, Huttenhofer A, Stadler PF: Mapping of conserved RNA secondary structures predicts thousands of functional noncoding RNAs in the human genome. Nat Biotechnol 2005, 23(11):1383-1390.

83. Mignone F, Grillo G, Licciulli F, lacono M, Liuni S, Kersey PJ, Duarte J, Saccone C, Pesole G: UTRdb and UTRsite: a collection of sequences and regulatory motifs of the untranslated regions of eukaryotic mRNAs. Nucleic Acids Res 2005, 33 Database: D141-146.

84. Mituyama T, Yamada K, Hattori E, Okida H, Ono Y, Terai G, Yoshizawa A, Komori T, Asai K: The Functional RNA Database 3.0: databases to support mining and annotation of functional RNAs. Nucleic Acids Res 2009, , 37 Database: D89-92.

85. Gardner PP, Daub J, Tate JG, Nawrocki EP, Kolbe DL, Lindgreen S, Wilkinson AC, Finn RD, Griffiths-Jones S, Eddy SR, et al: Rfam: updates to the RNA families database. Nucleic Acids Res 2009, , 37 Database: D136-140.

86. Washietl S, Hofacker IL, Stadler PF: Fast and reliable prediction of noncoding RNAs. Proc Natl Acad Sci USA 2005, 102(7):2454-2459.

87. Pedersen JS, Bejerano G, Siepel A, Rosenbloom K, Lindblad-Toh K, Lander ES, Kent J, Miller W, Haussler D: Identification and classification of conserved RNA secondary structures in the human genome. PLOS Comput Biol 2006, 2(4):e33.

88. Ahituv N, Prabhakar S, Poulin F, Rubin EM, Couronne O: Mapping cisregulatory domains in the human genome using multi-species conservation of synteny. Hum Mol Genet 2005, 14(20):3057-3063.

89. Bejerano G, Pheasant M, Makunin I, Stephen S, Kent WJ, Mattick JS, Haussler D: Ultraconserved elements in the human genome. Science 2004 304(5675):1321-1325.

90. Sandelin A, Bailey P, Bruce S, Engstrom PG, Klos JM, Wasserman WW, Ericson J, Lenhard B: Arrays of ultraconserved non-coding regions span the loci of key developmental genes in vertebrate genomes. BMC Genomics 2004, 5(1):99.

91. Woolfe A, Goodson M, Goode DK, Snell P, McEwen GK, Vavouri T, Smith SF, North P, Callaway H, Kelly K, et al: Highly conserved non-coding sequences are associated with vertebrate development. PLoS Biol 2005, 3(1):e7.

92. Sun H, Skogerbo G, Zheng X, Liu W, Li Y: Genomic regions with distinct genomic distance conservation in vertebrate genomes. BMC Genomics 2009, 10:133.

93. Pennacchio LA, Ahituv N, Moses AM, Prabhakar S, Nobrega MA, Shoukry M, Minovitsky S, Dubchak I, Holt A, Lewis KD, et al: In vivo enhancer analysis of human conserved non-coding sequences. Nature 2006, 444(7118):499-502.

94. Mesnard-Rouiller L, Bismuth J, Wakkach A, Poëa-Guyon S, Berrih-Aknin S: Thymic myoid cells express high levels of muscle genes. Journal of Neuroimmunology 2004, 148(1-2):97-105

95. Hubbard GB, Saphire DG, Hackleman SM, Silva MV, Vandeberg JL, Stone $\mathrm{WH}$ : Ontogeny of the thymus gland of a marsupial (Monodelphis domestica). Laboratory Animal Science 1991, 41(3):227-232.

96. Raviola E, Giuseppina R: Striated muscle cells in the thymus of reptiles and birds: An electron microscopic study. American Journal of Anatomy 1967, 121(3):623-645. 
97. Toro I, Olah I, Rohlich P, Viragh S: Electron microscopic observations on myoid cells of the frog's thymus. Anat Rec 1969, 165(3):329-341.

98. Gilmore RS, Bridges JB: Histological and ultrastructural studies on the myoid cells of the thymus of the domestic fowl, Gallus domesticus. J Anat 1974, 118(Pt 3):409-416.

99. Le Panse R, Berrih-Aknin S: Thymic myoid cells protect thymocytes from apoptosis and modulate their differentiation: implication of the ERK and Akt signaling pathways. Cell Death and Differentiation 2005, 12(5):463-472.

100. Lambropoulou M, Tamiolakis D, Venizelos I, Alexiadis G, Limberis V, Galazios G, Tsikouras P, Karamanidis D, Koutsougeras G, Nikolaidou S, et al: A stromal myoid cell line provokes thymic T-cell immigration at the second and third gestational trimesters. Rev Med Chir Soc Med Nat las 2007, 111(3):710-716

101. Oka T, Hayashi K, Nakaoka Y, Ohtsuki Y, Akagi T: Differentiation of rat thymic myoid progenitor cell line established by coculture with human T-lymphotropic virus type-I producing human T cells. Cell Tissue Res 2000, 300(1):119-127.

102. MacLennan DH, Rice WJ, Green NM: The mechanism of Ca2+ transport by sarco(endo)plasmic reticulum Ca2+-ATPases. The Journal of Biological Chemistry 1997, 272(46):28815-28818.

103. Gordon AM, Homsher E, Regnier M: Regulation of contraction in striated muscle. Physiological Reviews 2000, 80(2):853-924.

104. Villaseñor J, Besse W, Benoist C, Mathis D: Ectopic expression of peripheral-tissue antigens in the thymic epithelium: probabilistic, monoallelic, misinitiated. Proceedings of the National Academy of Sciences of the United States of America 2008, 105(41):15854-15859.

105. Derbinski J, Gäbler J, Brors B, Tierling S, Jonnakuty S, Hergenhahn M Peltonen L, Walter J, Kyewski B: Promiscuous gene expression in thymic epithelial cells is regulated at multiple levels. The Journal of Experimental Medicine 2005, 202(1):33-45.

106. Gotter J, Brors B, Hergenhahn M, Kyewski B: Medullary Epithelial Cells of the Human Thymus Express a Highly Diverse Selection of Tissue-specific Genes Colocalized in Chromosomal Clusters. J Exp Med 2004, 199(2):155-166.

107. Lannergren J, Elzinga G, Stienen GJ: Force relaxation, labile heat and parvalbumin content of skeletal muscle fibres of Xenopus laevis. J Physiol 1993, 463:123-140.

108. Muntener M, Kaser L, Weber J, Berchtold MW: Increase of skeletal muscle relaxation speed by direct injection of parvalbumin CDNA. Proc Natl Acad Sci USA 1995, 92(14):6504-6508.

109. Palmisano WA, Henzl MT: Molecular cloning of the thymus-specific parvalbumin known as avian thymic hormone: isolation of a full length CDNA and expression of the recombinant protein in Escherichia coli. Arch Biochem Biophys 1991, 285(2):211-220.

110. Murthy KK, Ragland WL: Immunomodulation by thymic hormones: studies with an avian thymic hormone. Prog Clin Biol Res 1984, 161:481-491.

111. Hapak RC, Zhao H, Boschi JM, Henzl MT: Novel avian thymic parvalbumin displays high degree of sequence homology to oncomodulin. J Biol Chem 1994, 269(7):5288-5296.

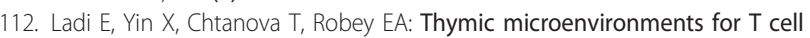
differentiation and selection. Nat Immunol 2006, 7(4):338-343.

113. Grounds MD, Garrett KL, Beilharz MW: The transcription of MyoD1 and myogenin genes in thymic cells in vivo. Experimental Cell Research 1992, 198(2):357-361.

114. Chan AS: Ultrastructure of myoid cells in the chick thymus. British Poultry Science 1995, 36(2):197-203

115. Venanzi ES, Melamed R, Mathis D, Benoist C: The variable immunological self: genetic variation and nongenetic noise in Aire-regulated transcription. Proceedings of the National Academy of Sciences of the United States of America 2008, 105(41):15860-15865

116. Anderson MS, Venanzi ES, Klein L, Chen Z, Berzins SP, Turley SJ, von Boehmer H, Bronson R, Dierich A, Benoist C, et al: Projection of an immunological self shadow within the thymus by the aire protein. Science (New York, NY) 2002, 298(5597):1395-1401.

117. Light N, Champion AE: Characterization of muscle epimysium, perimysium and endomysium collagens. Biochemical Journal 1984 219(3):1017-1026.

118. Deane EM, Cooper DW: Immunological development of pouch young marsupials. In The developing marsupial Models for biomedical research Edited by: Tyndale-Biscoe CH, Janssens PA. Berlin: Springer; 1988:190-199.
119. Wu TD, Watanabe CK: GMAP: a genomic mapping and alignment program for mRNA and EST sequences. Bioinformatics 2005, 21(9):1859-1875

120. Altschul SF, Madden TL, Schaffer AA, Zhang J, Zhang Z, Miller W, Lipman DJ: Gapped BLAST and PSI-BLAST: a new generation of protein database search programs. Nucleic Acids Res 1997, 25(17):3389-3402.

121. R: A Language and Environment for Statistical Computing. [http://www. R-project.org].

122. Benjamini $Y$, Hochberg $Y$ : Controlling the false discovery rate-A practical and powerful approach to multiple testing. Journal of the royal statistical society series B-methodological 1995, 57(1):289-300.

123. Huang DW, Sherman BT, Lempicki RA: Systematic and integrative analysis of large gene lists using DAVID bioinformatics resources. Nat Protoc 2009, 4(1):44-57.

124. Bauer S, Grossmann S, Vingron M, Robinson PN: Ontologizer 2.0-a multifunctional tool for $\mathrm{GO}$ term enrichment analysis and data exploration. Bioinformatics 2008, 24(14):1650-1651.

125. Ohta T: Evolution of gene families. Gene 2000, 259(1-2):45-52

126. Huang X, Madan A: CAP3: A DNA sequence assembly program. Genome Res 1999, 9(9):868-877.

doi:10.1186/1471-2164-12-420

Cite this article as: Wong et al:: Transcriptomic analysis supports similar functional roles for the two thymuses of the tammar wallaby. BMC Genomics 2011 12:420.

\section{Submit your next manuscript to BioMed Central and take full advantage of:}

- Convenient online submission

- Thorough peer review

- No space constraints or color figure charges

- Immediate publication on acceptance

- Inclusion in PubMed, CAS, Scopus and Google Scholar

- Research which is freely available for redistribution
Ciomed Central 